\title{
Measurement of the Sivers asymmetry at SpinQuest experiment
}

Ching Him Leung

University of Illinois at Urbana Champaign

New Perspectives 2021

16-19 August 2021

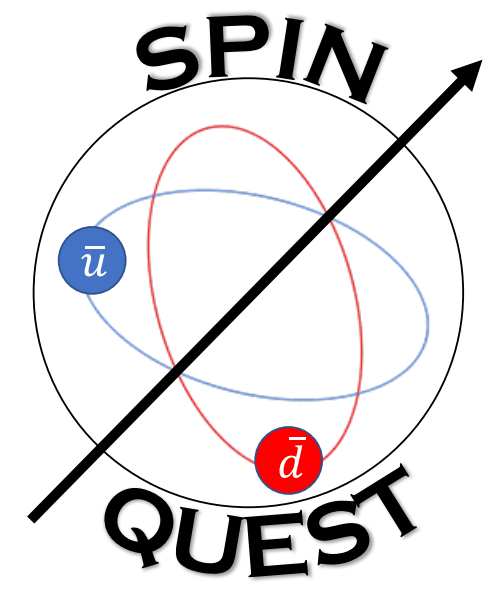




\section{E1039/SpinQuest}

- A fixed target experiment with a transversely polarized $\mathrm{NH}_{3}$ and $\mathrm{ND}_{3}$ targets

- Measure the azimuthal asymmetry in Drell-Yan process

- Provide information on the Sivers function of the light sea quarks

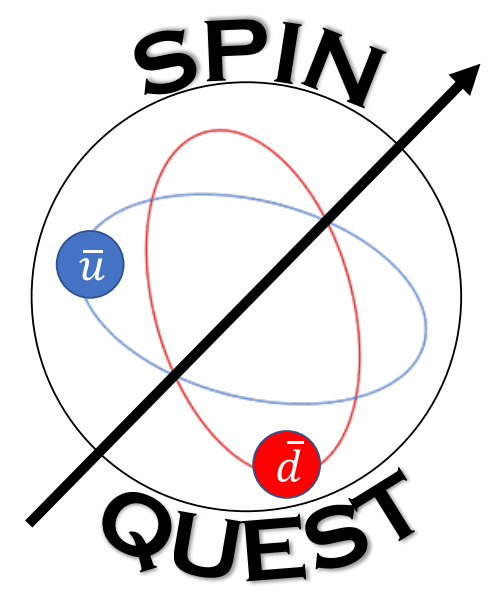




\section{Drell-Yan process}

- A long history of dimuon experiment here in Fermilab

- Previous experiments were addressing the longitudinal motion of quarks
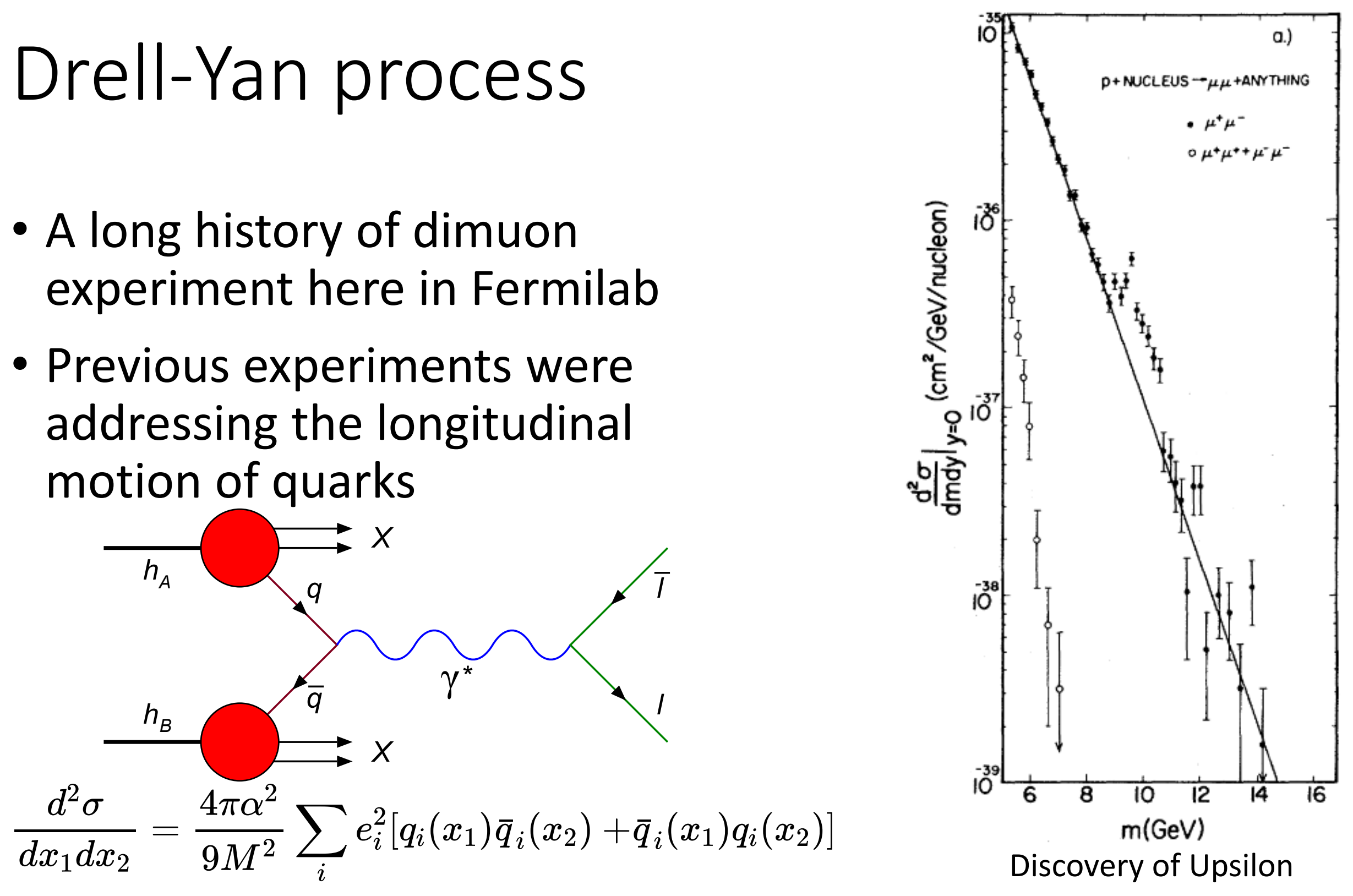

Phys. Rev. Lett. 


\section{Drell-Yan process}

- A long history of dimuon experiment here in Fermilab

- Previous experiments were addressing the longitudinal motion of quarks

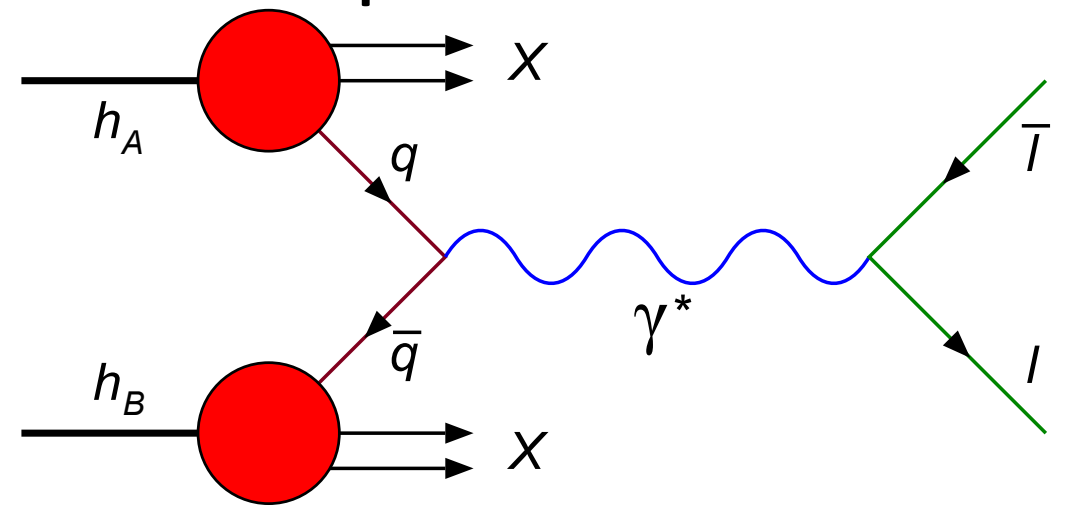

p A $\rightarrow \mu^{+} \mu^{-} \mathrm{X}$

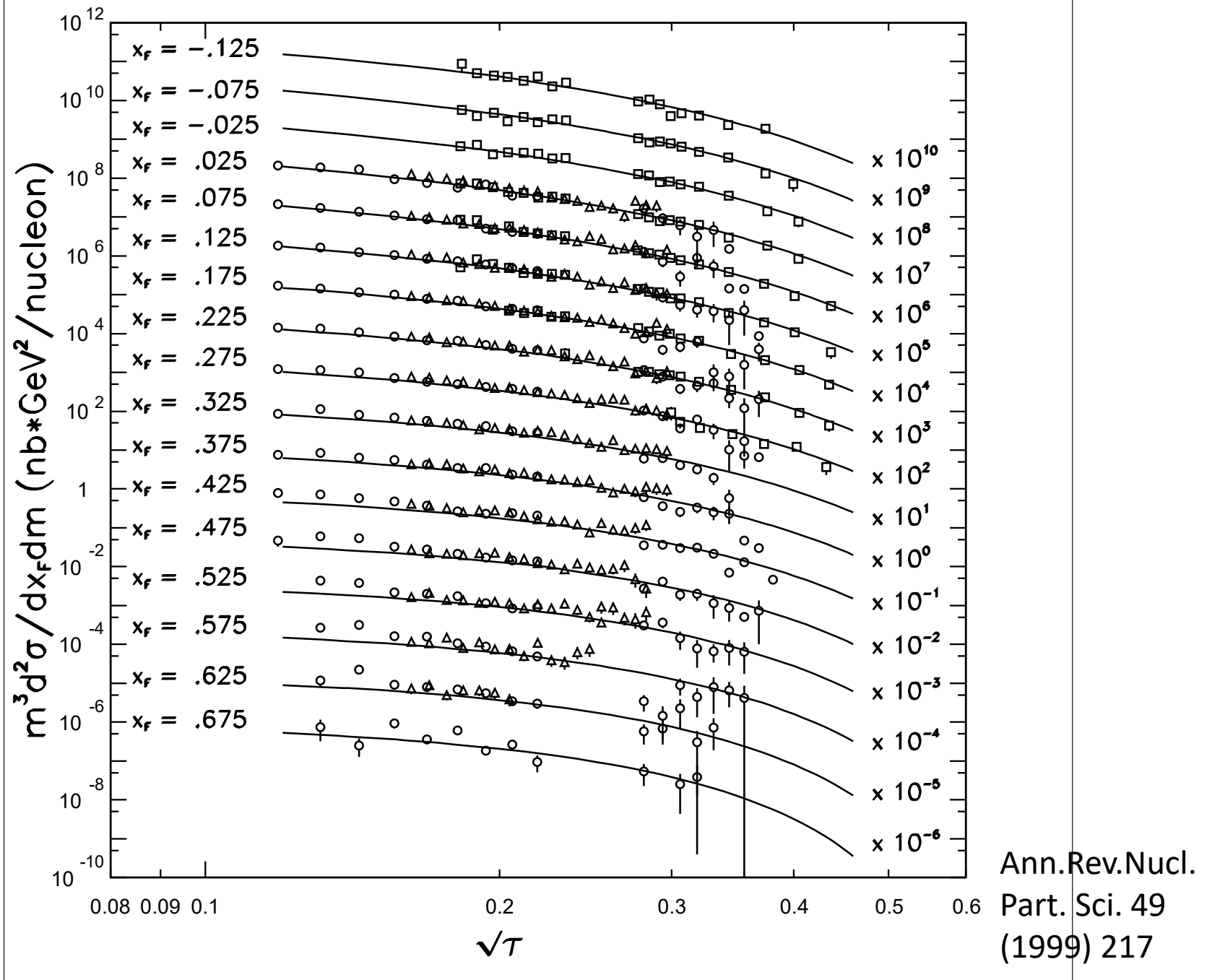

A compilation of Drell-Yan data from CERN(NA3) and Fermilab(E605 and E772) 


\section{SeaQuest Experiment}

- Most recent Drell-Yan Experiment at Fermilab

- $120 \mathrm{GeV}$ proton beam from Main Injector

- A forward spectrometer

- The antiquarks are more likely to come from the target

- Design to measure the sea quark structure at higher $x$

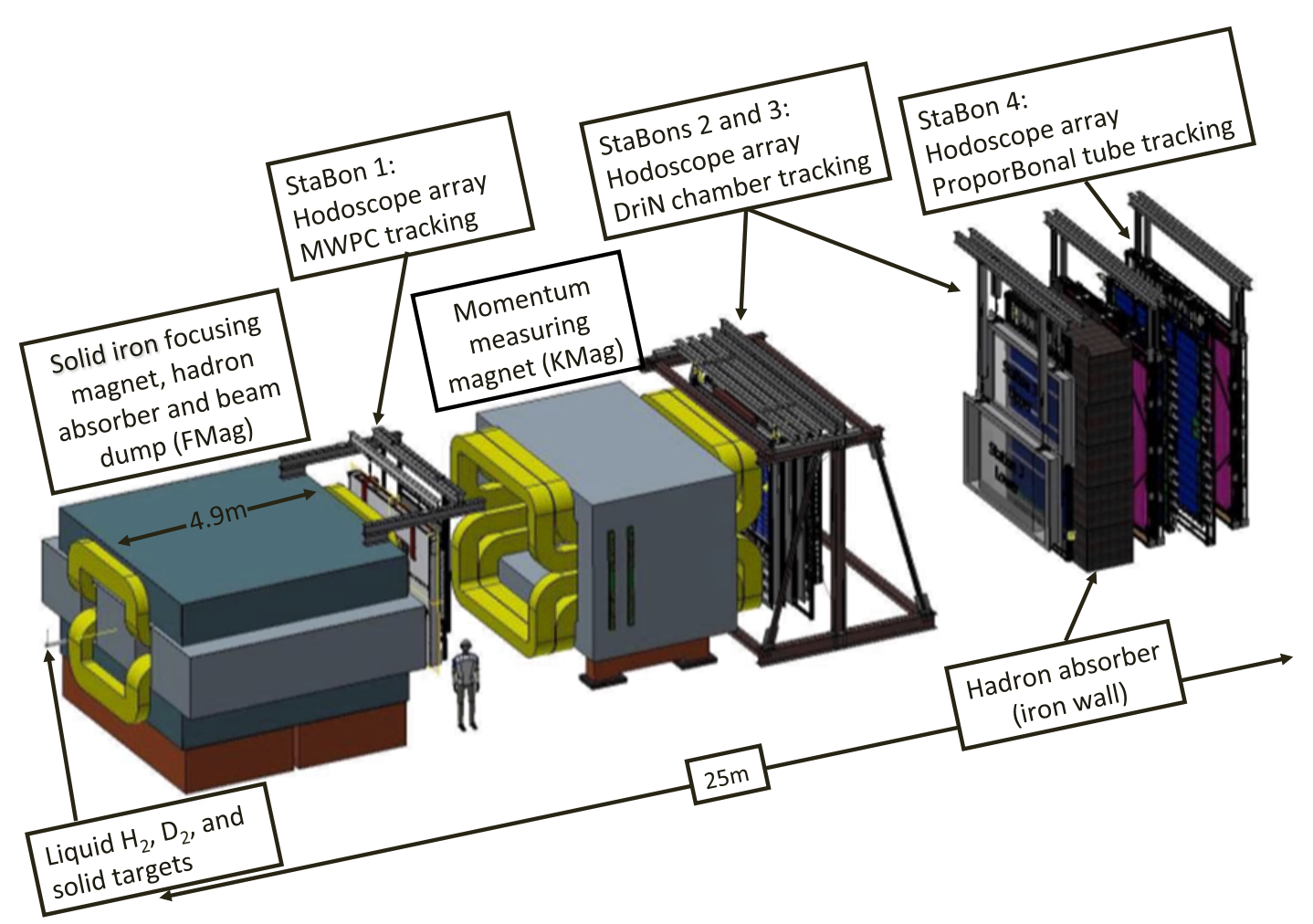




\section{Result from SeaQuest}

- The mass spectrum contain the $J / \psi$ peak and well as the Drell-Yan continuum at high mass

- The antiquark structure can be probed using the Drell-Yan process

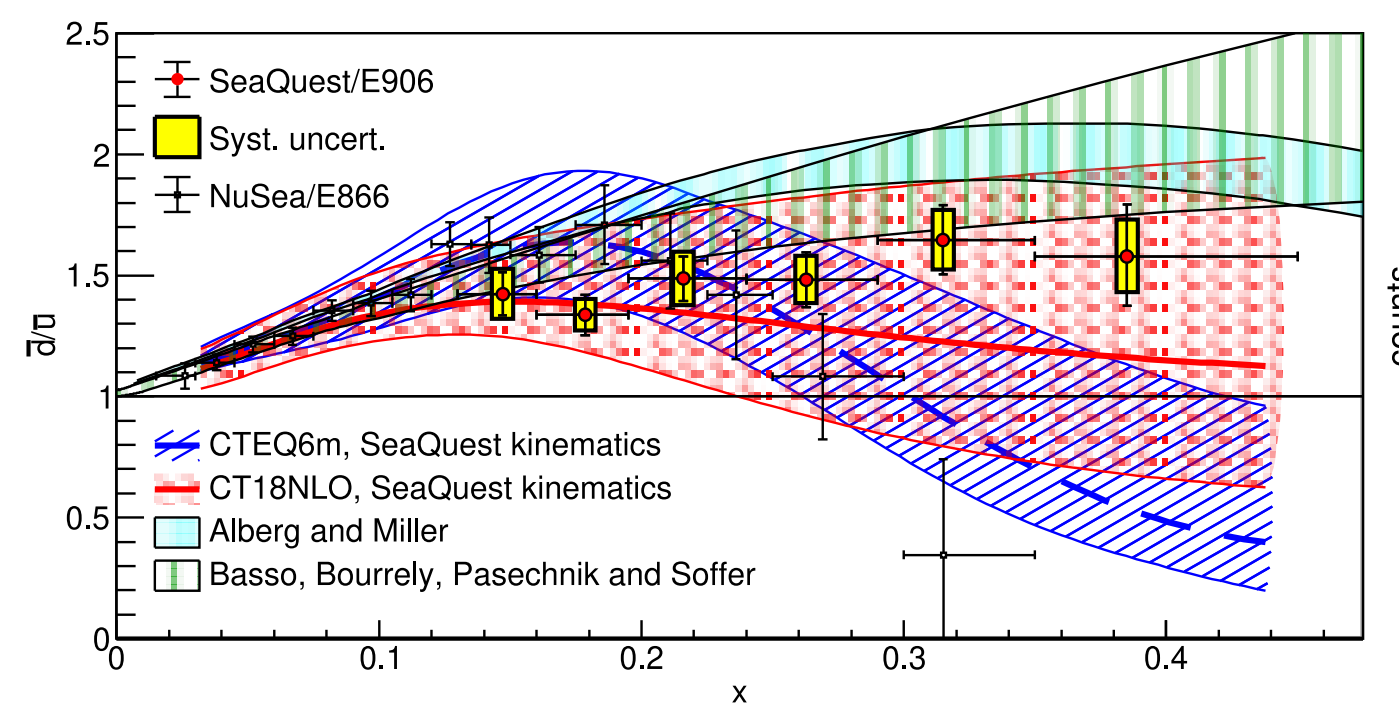

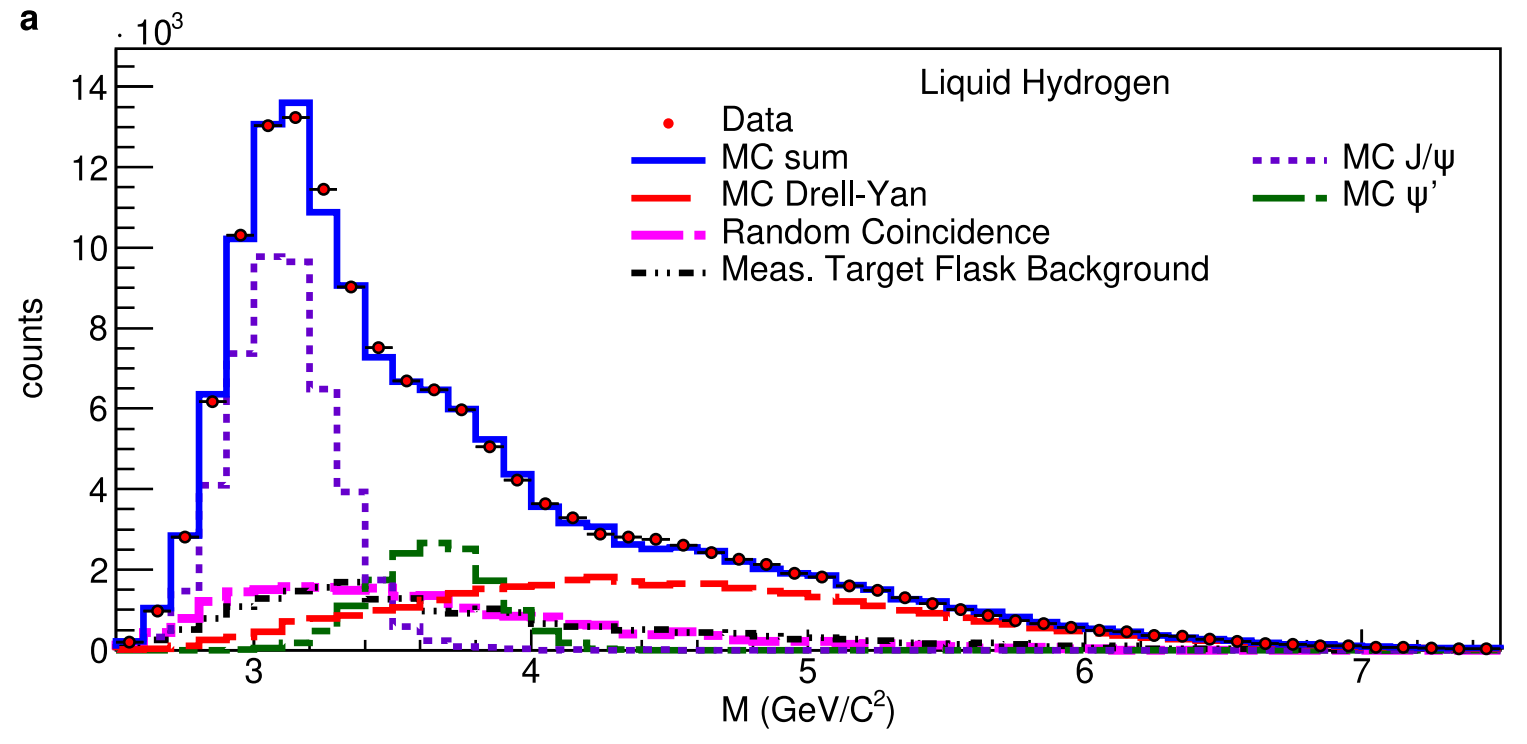

Nature volume 590, pages 561-565 (2021) 


\section{SpinQuest Experiment}

- First Drell-Yan experiment at Fermilab with a polarized target

- The same spectrometer from SeaQuest will be used

- The transversely polarized target allows us to probe the transverse degree of freedom of partons in nucleon
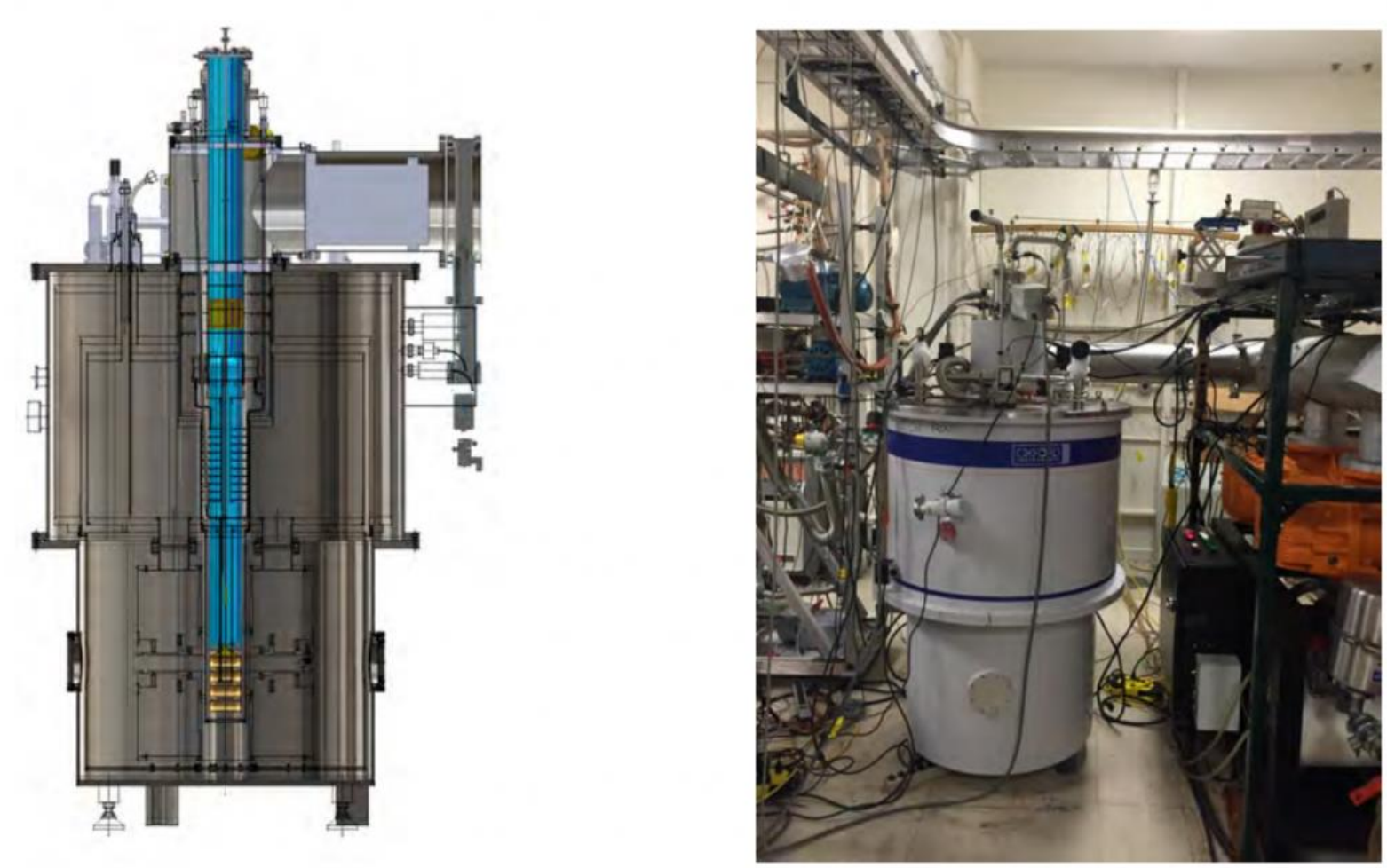


\section{Transverse momentum dependent parton distributions (TMDs)}

- Eight quark TMDs

Leading Twist TMDs

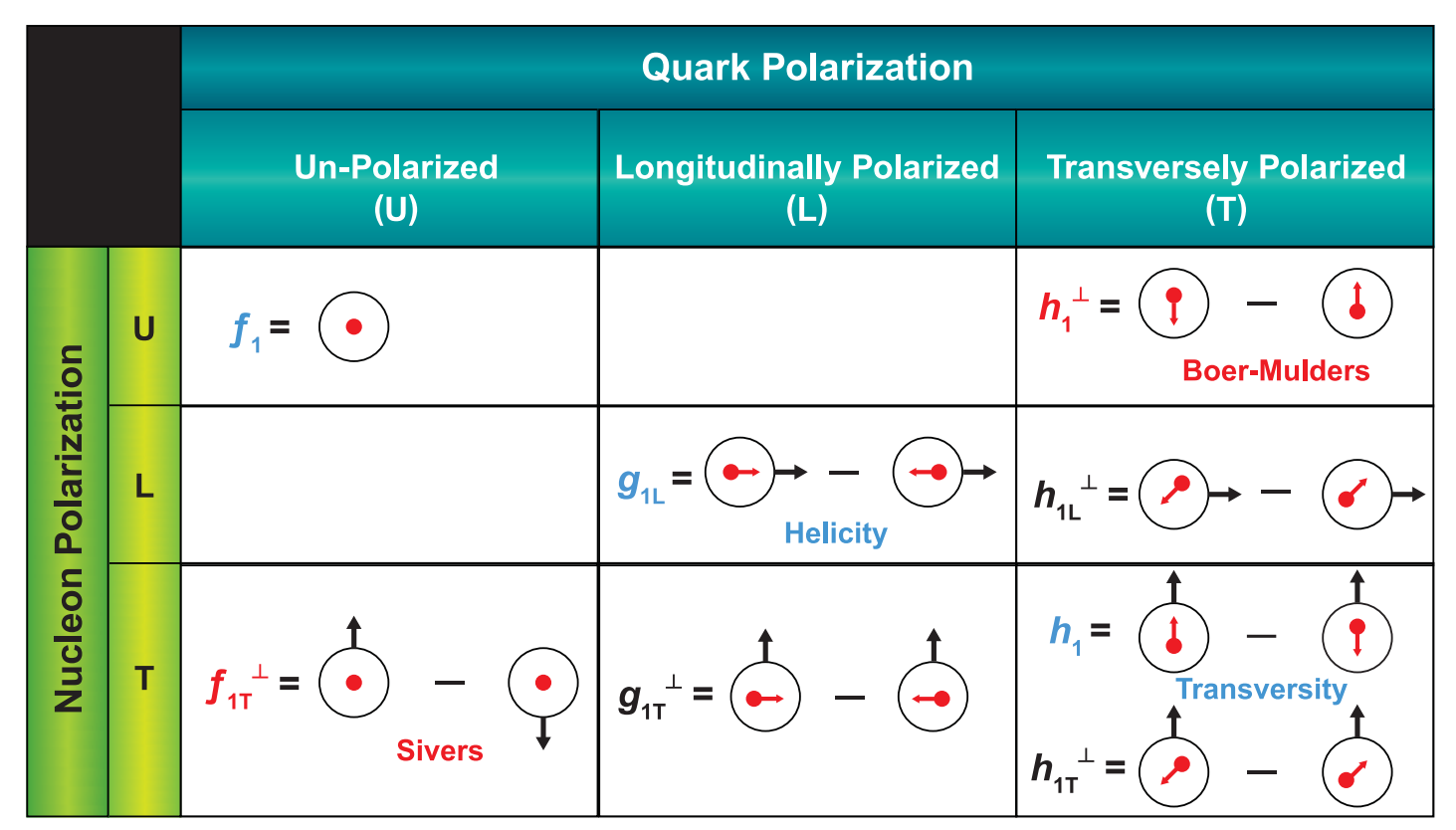

- Classified by the polarization of the nucleon and quarks

- Similar TMDs for gluon 


\section{Transverse momentum dependent parton distributions (TMDs)}

- Eight quark TMDs

Leading Twist TMDs

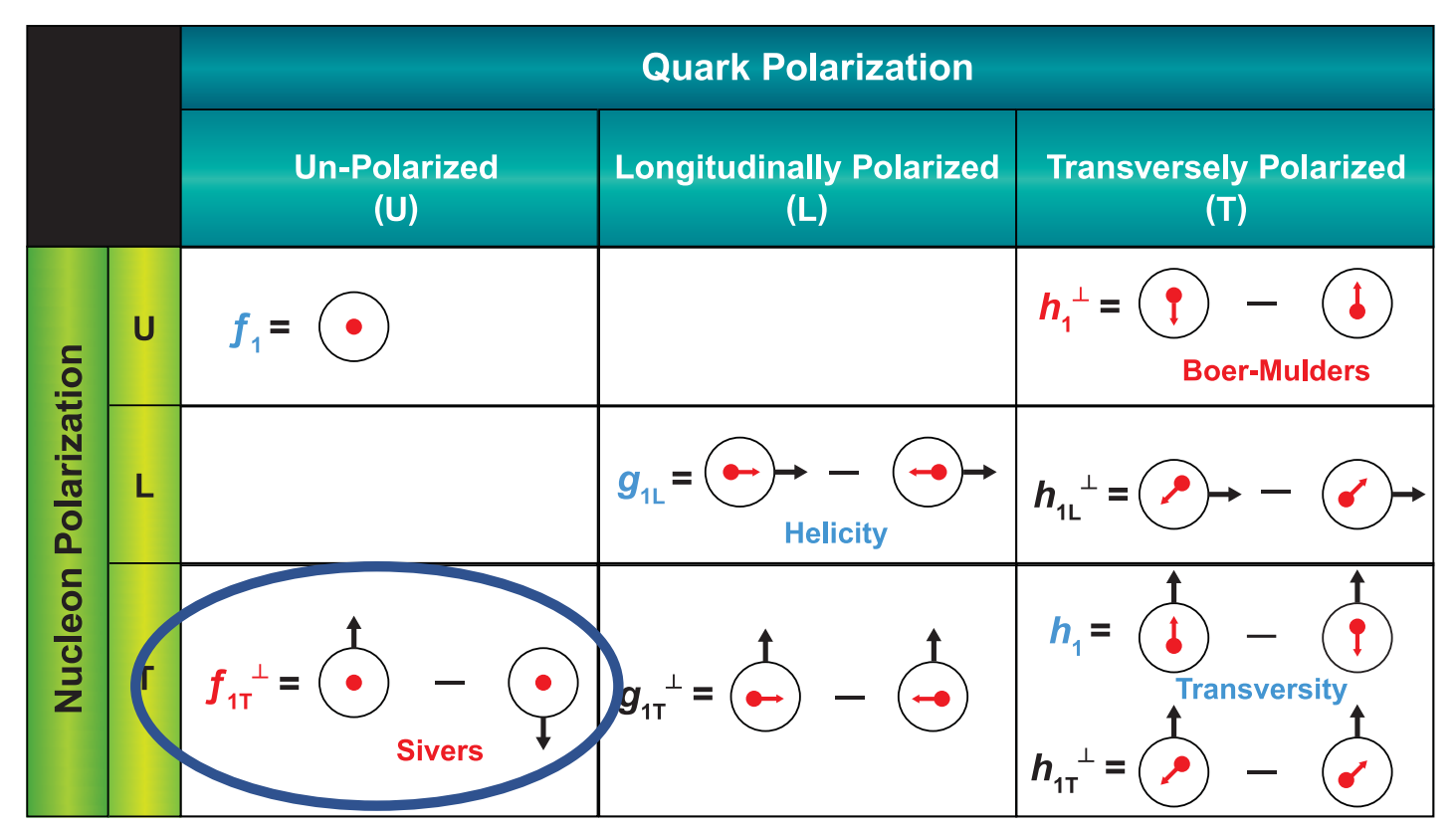

- Classified by the polarization of the nucleon and quarks

- Similar TMDs for gluon 


\section{Sivers function}

- Correlation between the quark transverse momentum and the nucleon transverse spin vector

- Causes a left-right asymmetry in measured dimuon distribution relative to nucleon transverse spin direction

- The current models are fitted to DIS data

- Mostly sensitive to the valence quarks

$$
\hat{f}_{q / p^{\uparrow}}\left(x, \vec{k}_{\perp}\right)=f_{q / p}\left(x, k_{\perp}\right)+\frac{2 k_{\perp}}{m_{p}} f^{\perp q}\left(x, k_{\perp}\right) \vec{S} \cdot\left(\hat{P} \times \hat{k}_{\perp}\right)
$$

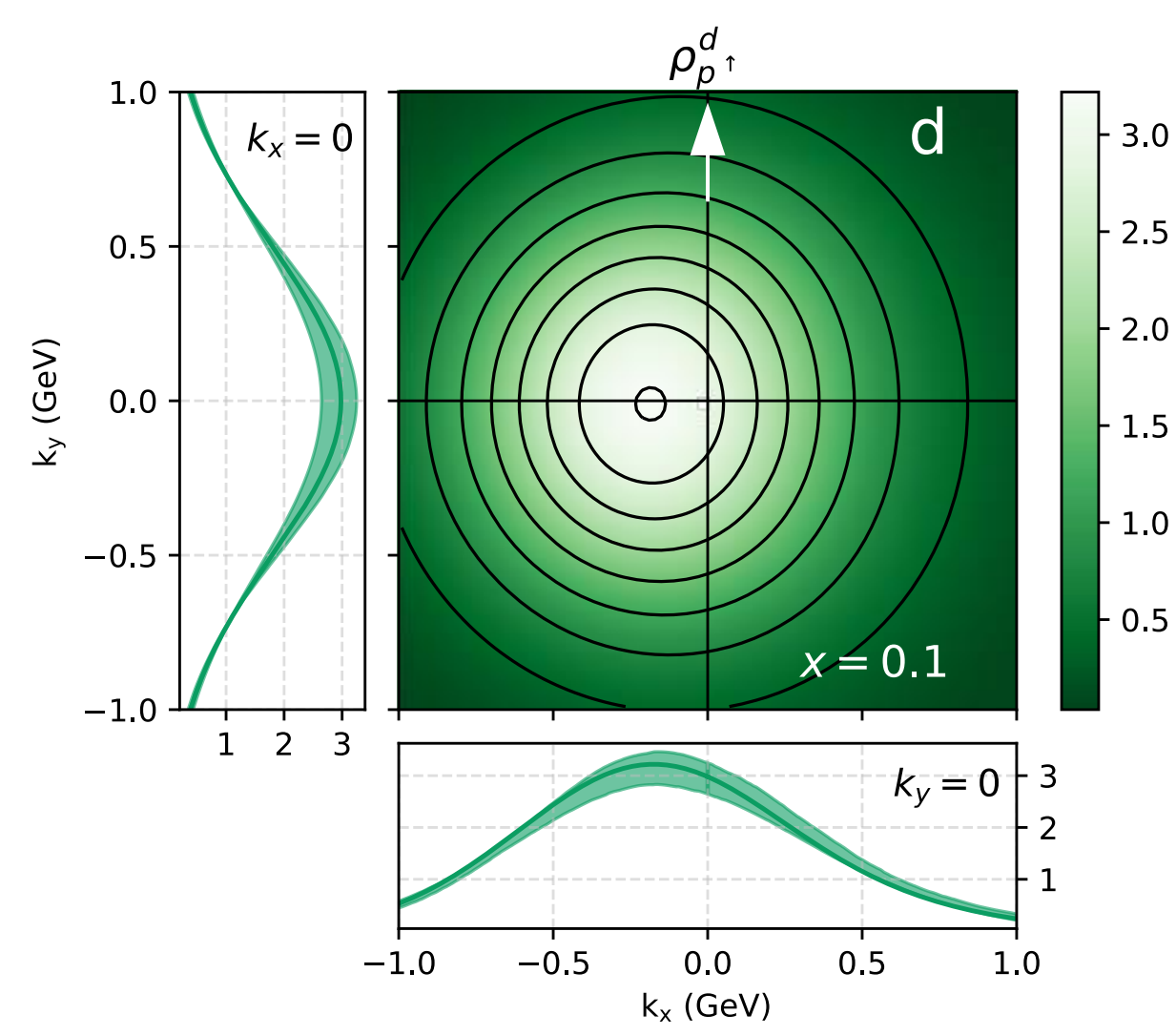

The number density of quarks in a proton polarized along $\hat{y}$, for $x=0.1$ arXiv:2004.14278v1 


\section{Measuring Sivers functions in the Drell-Yan process}

- The angular distribution can be expressed as

$$
\begin{aligned}
\frac{d \sigma}{d q^{4} d \Omega} \propto & \hat{\sigma}_{U}\left(1+S_{T} D_{1} A_{T}^{\sin \varphi_{s}} \sin \varphi_{s}\right. \\
& +D_{2}\left[A_{T}^{\sin \left(2 \varphi_{C S}-\varphi_{S}\right)} \sin \left(2 \varphi_{C S}-\varphi_{S}\right)\right. \\
& \left.\left.\left.+A_{T}^{\sin \left(2 \varphi_{C S}+\varphi_{S}\right)} \sin \left(2 \varphi_{C S}+\varphi_{S}\right)\right]\right\}\right)
\end{aligned}
$$

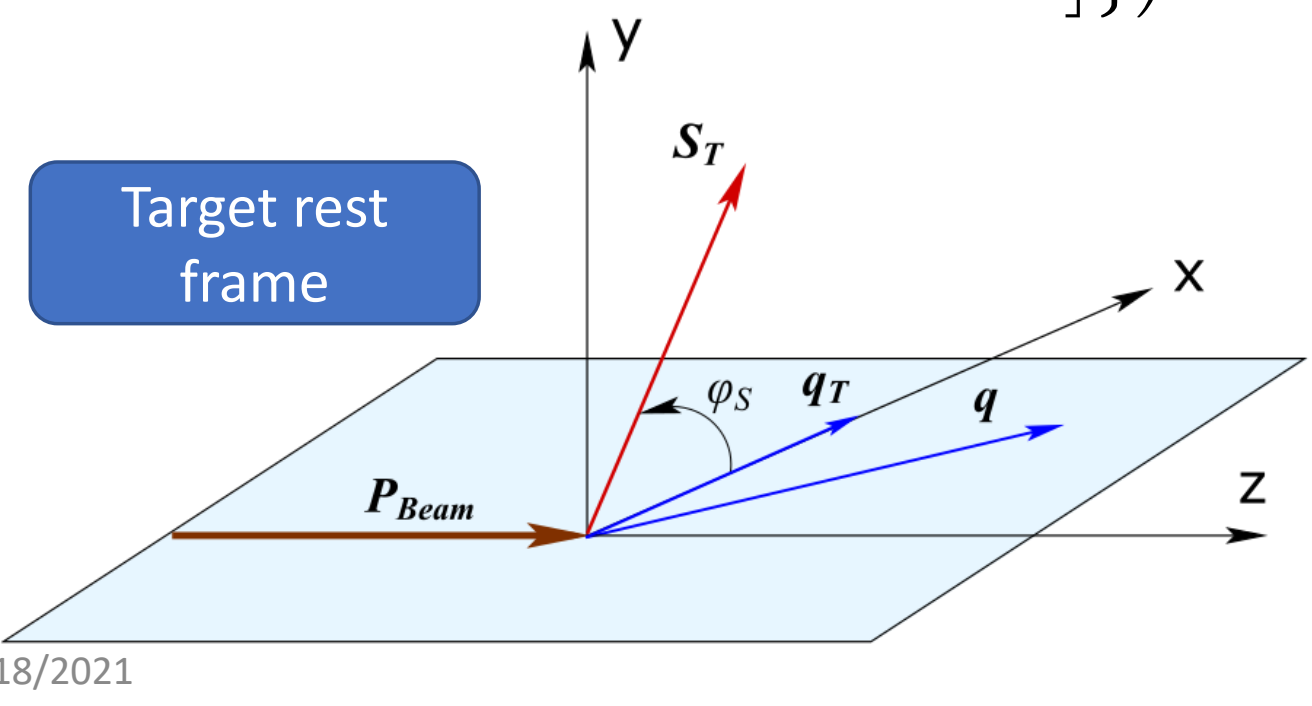

- $A_{T}^{\sin \varphi_{S}}$ is related to the convolution of the Sivers function $f_{1 T}^{\perp}$ and unpolarized TMD $f_{1}$

- The other modulations are related to other TMDs 


\section{Expected Sensitivity}

- Expected results after two years of combined running on $\mathrm{NH} 3$ and ND3 targets.

- The three bands are from different theoretical predictions

- Fitted to DIS data, insensitive to the sea quark contribution

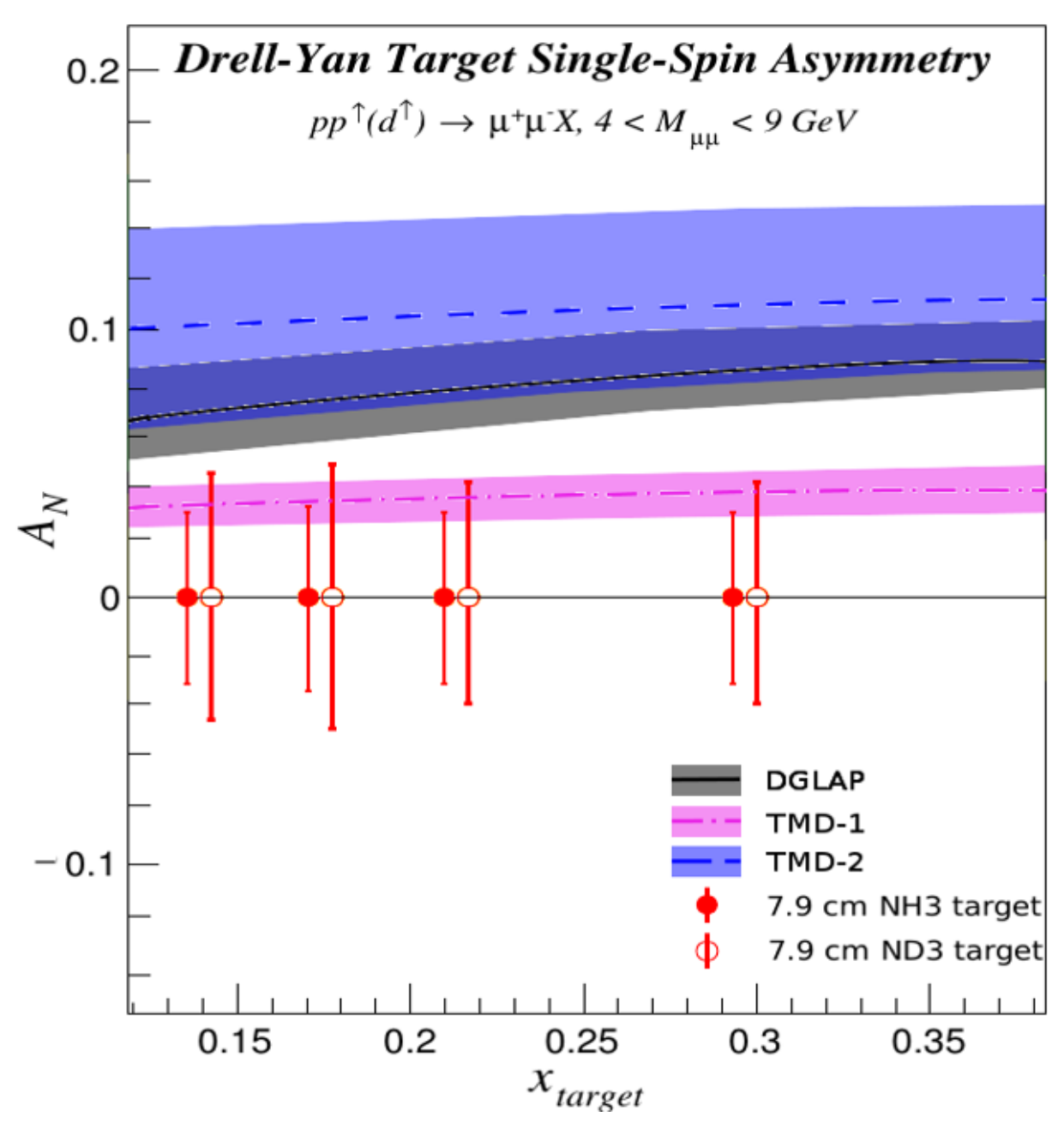

DGLAP: M. Anselmino et al arXiv:1612.06413

TMD-1: M. G. Echevarria et al arXiv:1401.5078

TMD-2: P. Sun and F. Yuan arXiv:1308.5003 


\section{$J / \psi$ production}

- The $J / \psi$ production is sensitive to the gluon Sivers function as well as the quark Sivers function

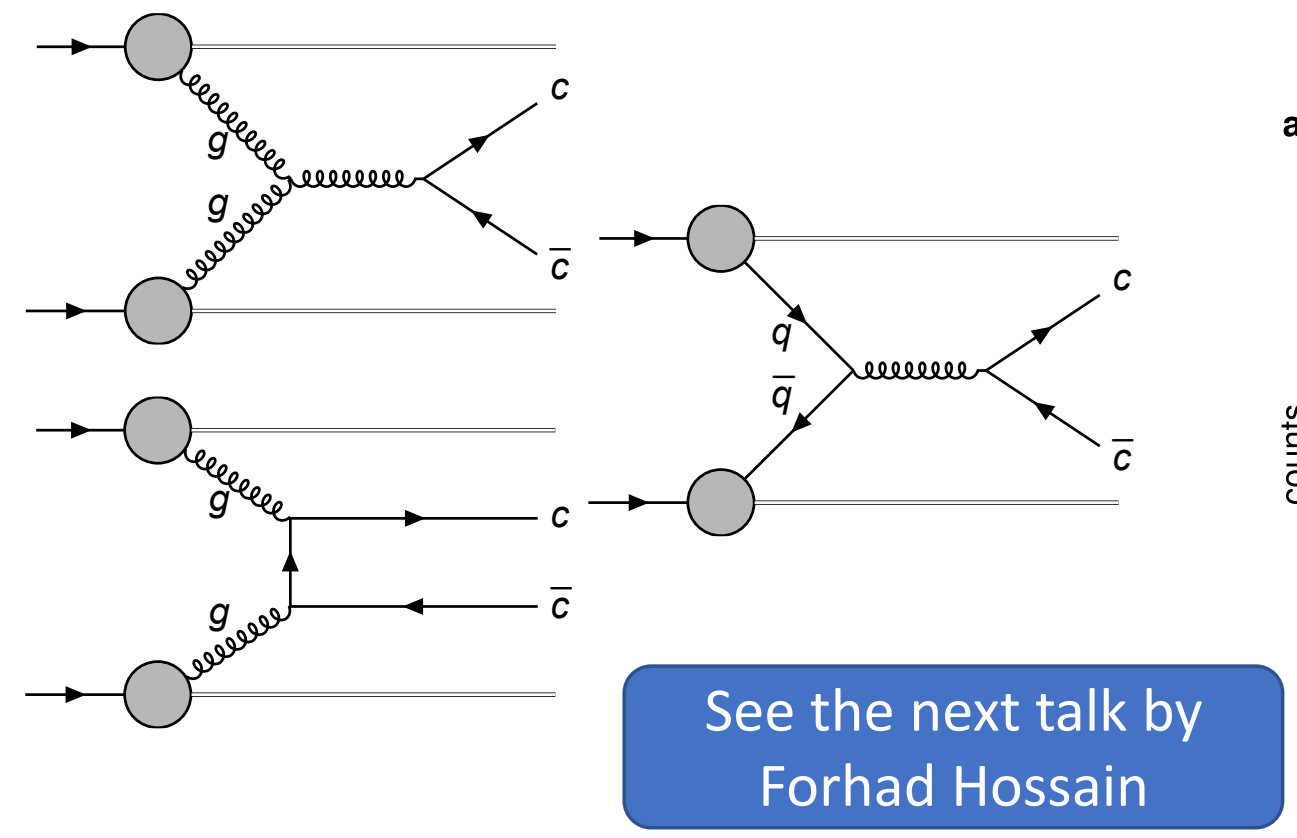

$\mathrm{NRQCD} p+p \rightarrow J / \Psi$ at $\mathrm{E}_{\text {beam }}=120 \mathrm{GeV}$

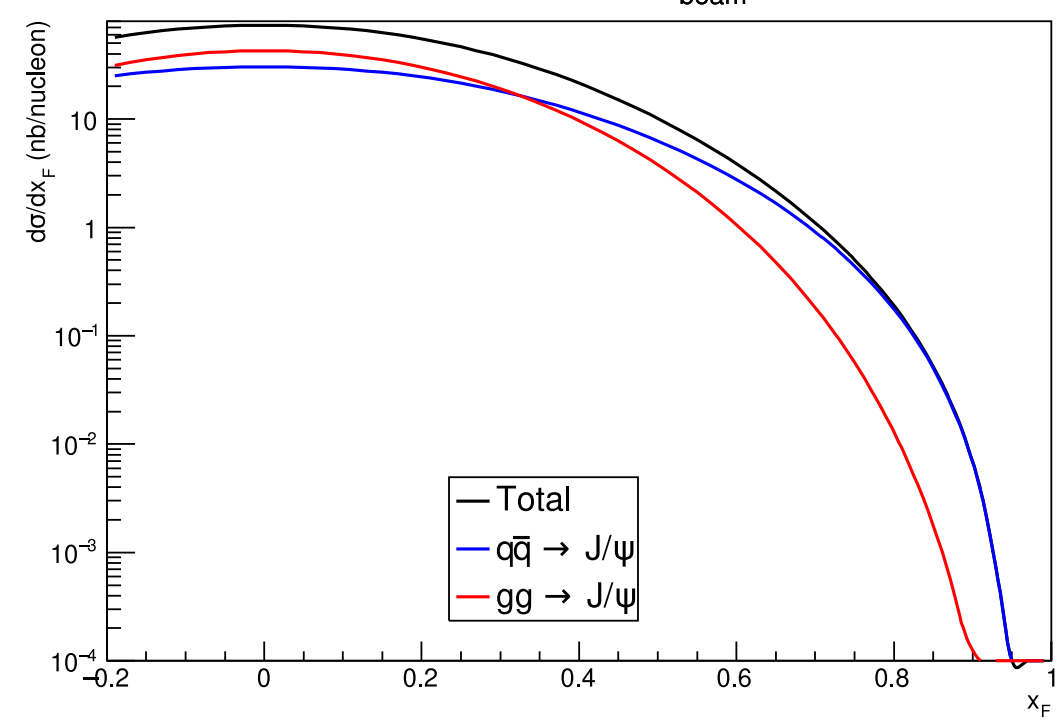

a

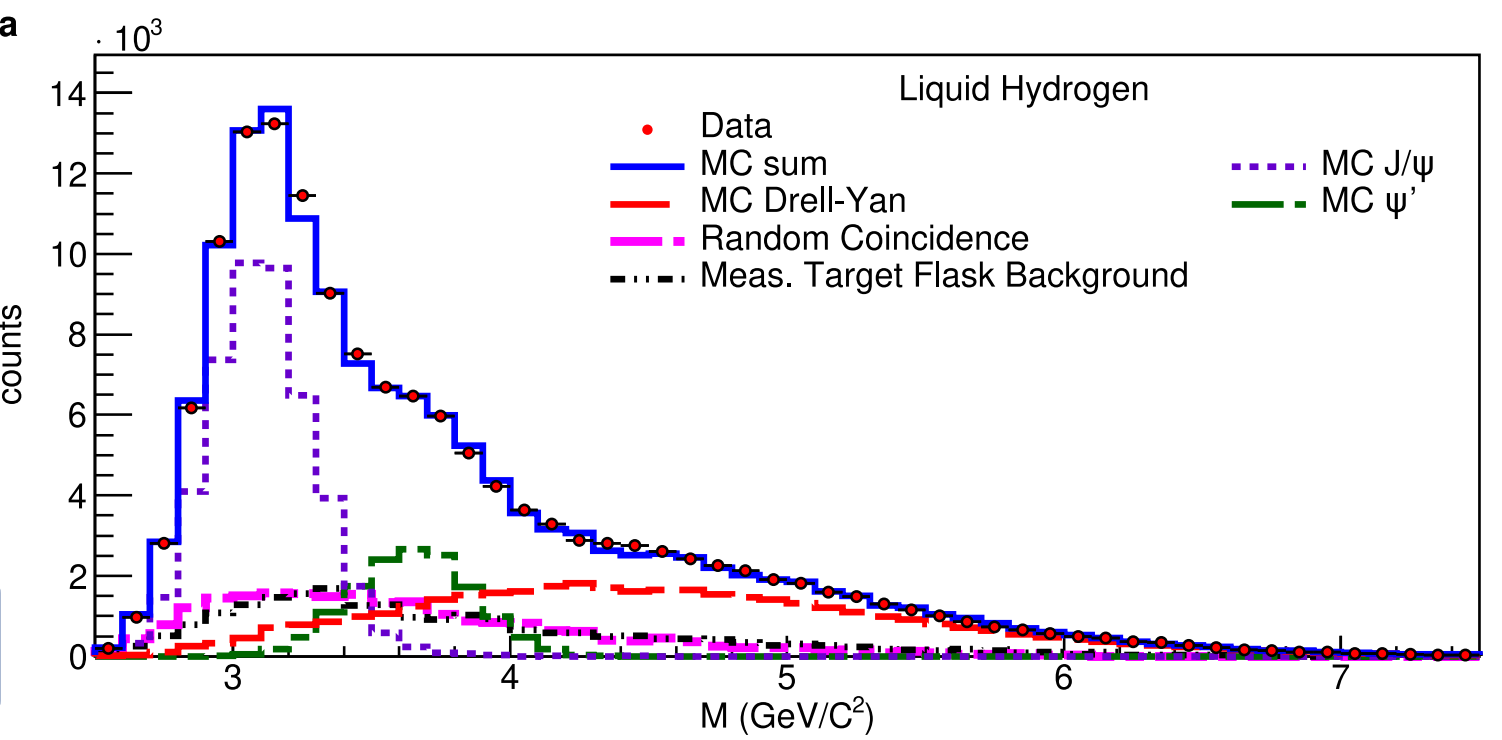




\section{Conclusion}

- SpinQuest can measure the transverse single spin asymmetry in DrellYan process and charmonium production

- This can provide information to the Sivers function for the quarks and gluons

- Expect to start beam commissioning in December 2021 and physics run in spring 2022 


\section{Orbital angular momentum}

- The spin of the quark alone cannot fully account for the spin of the proton

- Orbital angular momentum of the sea quarks can be significant contribution

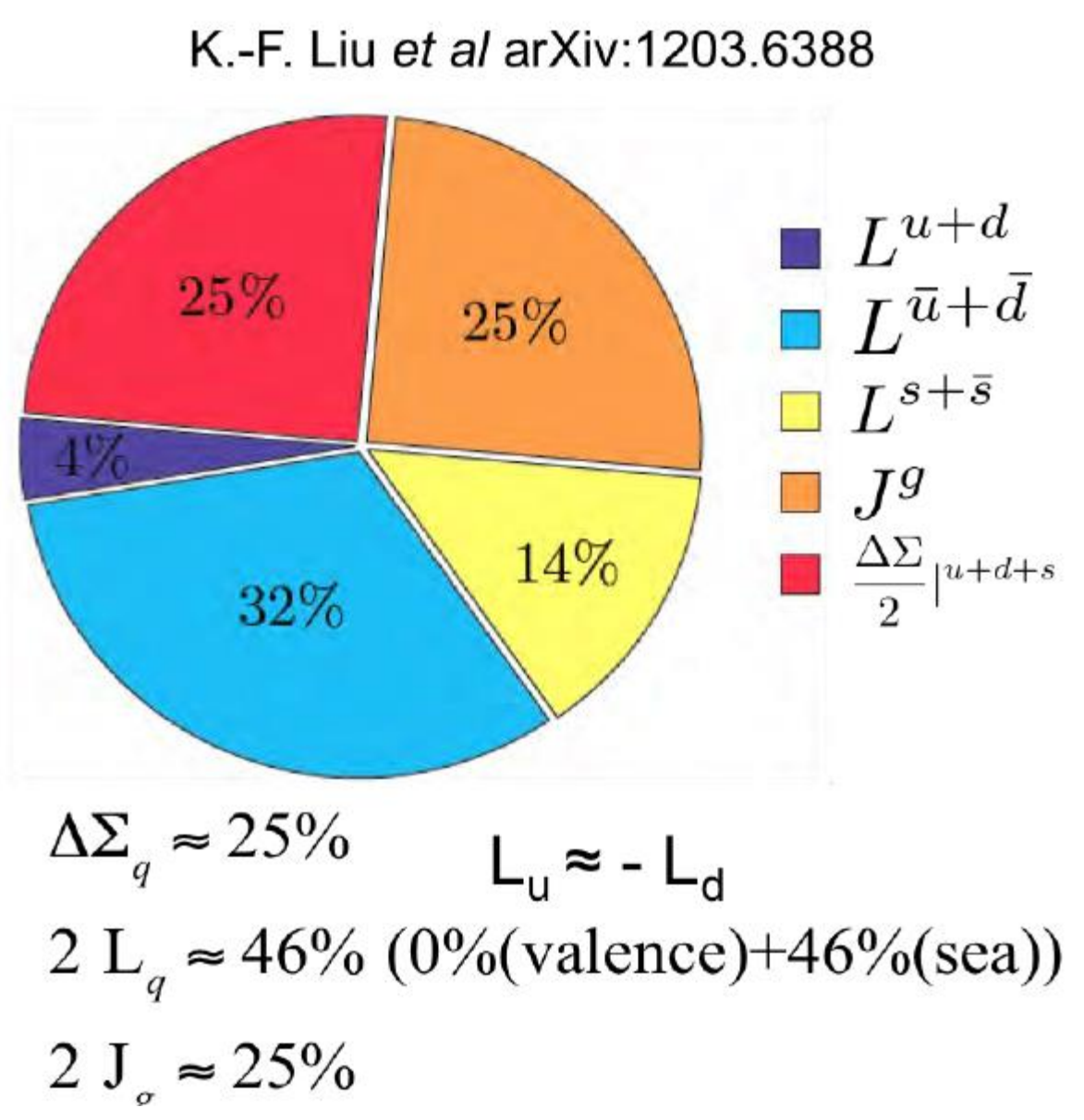

